\title{
Psicologia positiva e o estudo do bem-estar subjetivo
}

\section{Positive psychology and the subjective well-being study}

\author{
Paola Moura PASSARELI \\ José Aparecido da SILVA²
}

\begin{abstract}
Resumo
O presente artigo visou apresentar o surgimento da Psicologia Positiva como um importante novo campo de estudos da Psicologia contemporânea, em um momento em que novos estudos têm focado a compreensão das forças e virtudes humanas. Especial atenção é dada a um de seus principais componentes - o bem-estar subjetivo -, também conhecido como felicidade. Buscando elucidar os aspectos envolvidos no estudo do bem-estar subjetivo, este artigo procurou abordar alguns de seus principais correlatos. O melhor entendimento dos fatores envolvidos com o surgimento tanto de emoções positivas quanto de negativas permite uma maior compreensão da condição humana diante das adversidades.
\end{abstract}

Unitermos: emoções positivas; bem-estar subjetivo; psicologia positiva.

\begin{abstract}
The proposal of the this paperwas to present the emerging of the PositivePsychology as a new important field of the contemporary Psychology, in a moment when new studies has focused the understanding of human strengths and values. One of its main components gave special attention, subjective well-being, also known as happiness. Searching to elucidate the involved aspects in the study of subjective well-being, this article looked for to approach some of its most important correlates. The best agreement of the involved factors with the sprouting of positive and negative emotions allows a better understanding of the human being's condition up against the adversities.
\end{abstract}

Uniterms: positive affect; subjectivity; positive psychology.

A revista científica American Psychologist dedicou, no ano 2000, sua edição especial a um novo ramo de estudos da Psicologia, a Psicologia Positiva - o estudo da emoção positiva, do caráter positivo, e de instituições positivas (Seligman \& Csikszentmihalyi, 2000), reconhecendo a importância do desenvolvimento de pesquisas sobre esse tema.

O novo foco em estudos sobre as forças e as potencialidades humanas significa uma mudança em interesses concentrados apenas em emoções negativas, tão freqüentes em pesquisas empíricas até o presente momento, e o surgimento de um novo grupo de pesquisas interessadas em emoções positivas, até então realizadas de maneira muito escassa. Os estudos sobre pessoas infelizes são abundantes na literatura, enquanto são deixados de lado os aspectos positivos do potencial humano (Myers, 2000; Seligman, 2004).

Diener e Seligman (2002) vão mais além quando afirmam que os estudos sobre pessoas felizes são raros na literatura científica, enquanto investigações sobre

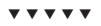

1 Universidade de São Paulo, Faculdade de Filosofia, Ciências e Letras de Ribeirão Preto, Programa de Pós-Graduação em Psicobiologia. Av. Bandeirantes, 3900, Bloco 6, Monte Alegre, 14040-901, Ribeirão Preto, SP, Brasil. Correspondência para/Correspondence to: P.M. PASSARELI. E-mail: <paola@hmtr.com.br>. Apoio: CAPES. 
pessoas muito felizes não existem. Segundo Myers e Diener (1995), artigos psicológicos referentes a estados negativos excedem aqueles que estudam estados positivos em uma proporção de 17 artigos para um. A diferença observada entre o número de artigos é surpreendente.

Considerando então a importância da Psicologia Positiva, faz-se necessária uma maior compreensão sobre o tema. Esse novo ramo da Psicologia pode ser definido como o estudo científico de emoções positivas, forças e virtudes humanas (Bacon, 2005; Seligman \& Csikszentmihalyi, 2000; Sheldon \& King, 2001).

Os achados das pesquisas envolvendo a Psicologia Positiva têm a intenção de suplementar, não remover ou substituir, o que se conhece sobre sofrimento, fraqueza e desordens humanas. A intenção é ter uma compreensão científica mais completa e mais equilibrada sobre as experiências humanas, e não considerar uma maior relevância de uma experiência sobre as outras (Seligman, Steen, Park \& Peterson, 2005).

Os estudos envolvendo a Psicologia Positiva convidam psicólogos de todas as orientações e interesses a se juntar à exploração do lado positivo da vida (Simonton \& Baumeister, 2005).

A Psicologia não deve se ater apenas ao estudo da doença, da fraqueza e das perdas; semelhante atenção deve ser dada ao estudo de forças e virtudes humanas. O tratamento psicológico não envolve apenas um reparo de algo que está quebrado, mas o cultivo do que há de melhor em cada indivíduo. A Psicologia não deve ser concebida apenas como uma filial da Medicina preocupada com a doença ou a saúde, ela é muito mais abrangente, pois envolve o trabalho, a educação, a introspecção, o amor e o crescimento (Seligman \& Csikszentmihalyi, 2000).

Em 2002, Seligman publicou o livro intitulado Authentic Happiness - traduzido para o português no ano de 2004 -, onde o autor relata suas reflexões sobre a Psicologia Positiva e sua relação com a felicidade. Para esse autor, a Psicologia Positiva teria três pilares. O primeiro seria o estudo da emoção positiva; o segundo, o estudo dos traços positivos, "principalmente as forças e as virtudes, mas também as habilidades, como a inteligência e a capacidade atlética", e o terceiro, "o estudo das instituições positivas, como a democracia, a

514 família e a liberdade, que dão suporte às virtudes que, por sua vez, apóiam as emoções positivas" (Seligman, 2004, p.13).

Seligman (2004, p.287) afirmou que ambos os termos - felicidade e bem-estar subjetivo -, que podem ser utilizados de maneira intercambiável, descrevem "os objetivos de todos os esforços da Psicologia Positiva", incluindo "sentimentos positivos (êxtase e conforto, por exemplo) e atividades positivas (absorção e dedicação, por exemplo)".

\section{O bem-estar subjetivo como componente da psicologia positiva}

O bem-estar subjetivo é um importante componente da Psicologia Positiva. É um aspecto que pode favorecer a maneira como vemos a nós mesmos e as outras pessoas, o que pode resultar em maior prazer em vivenciar as situações cotidianas e o relacionamento com nossos pares. Torna-se importante, cada vez mais, conhecer os aspectos relacionados a esse tema.

O bem-estar subjetivo, também chamado de felicidade (Diener, 2000; Seligman, 2004), pode ser nomeado de extroversão estável, parecendo o afeto positivo na felicidade estar relacionado à fácil sociabilidade, o que propicia uma interação natural e agradável com outras pessoas. Faz sentido, assim, comparar a felicidade com a extroversão (Francis, 1999).

Seligman (2004) afirmou que as pessoas felizes têm mais amigos, tanto amigos casuais como amigos íntimos, permanecem casadas por um maior período e participam de mais atividades de grupo. Todos esses fatores indicam uma facilitação de contatos sociais.

Pessoas com o bem-estar elevado parecem ter melhores relações sociais do que pessoas que apresentam o bem-estar rebaixado. Relações sociais positivas mostram-se necessárias para o bem-estar. Existem diferentes dados sugerindo que o bem-estar leva ao desenvolvimento de boas relações sociais e não é meramente seguido por elas (Diener \& Seligman, 2004). Muitos estudos têm indicado que, comparadas às pessoas solteiras, as pessoas casadas têm melhor saúde física e psicológica, além de viverem mais (Burman \& Margolin, 1992).

Confirmando esses estudos, evidências experimentais indicam que as pessoas tendem a apresentar sofrimento quando não fazem parte de nenhum tipo de grupo ou quando têm relações pobres dentro dos 
grupos a que pertencem (Diener \& Seligman, 2004). Verificou-se, assim, que participar de grupos, como grupos de amigos, de trabalho, de apoio, é um fator favorável para o bem-estar subjetivo.

Estudos indicam, por outro lado, que o aumento da renda produziria pouco benefício adicional ao bem-estar - fato já observado por estudos realizados em diferentes nações -, o que sugere uma baixa correlação entre indicadores econômicos e diferentes componentes e formas de bem-estar, como felicidade, satisfação de vida e afeto positivo (Diener \& Biswas-Diener, 2002).

Diener e Seligman (2004) relataram que nos últimos 50 anos os rendimentos aumentaram de forma constante nos Estados Unidos, triplicando a renda per capita, mas no mesmo período não se observou um crescimento proporcional de satisfação de vida.

Diener e Biswas-Diener (2002) sustentaram que uma renda mais elevada auxiliaria as avaliações de bem-estar subjetivo no caso de pessoas extremamente pobres, porém a partir de uma determinada renda não se verificaria a correlação entre aumento da riqueza e aumento do bem-estar subjetivo (Diener, 2004).

De fato, constata-se que felicidade, ou bem-estar subjetivo, não é apenas a ausência de depressão, mas também a presença de um número de emoções e estados cognitivos positivos (Joseph, Linley, Harwood, Lewis \& McCollam, 2004). Segundo Diener, Suh e Oishi (1997), o campo do bem-estar subjetivo inclui os estados indesejáveis tratados pelos psicólogos clínicos, embora não se limite apenas ao estudo desses estados indesejáveis. Para esses autores, o bem-estar subjetivo também se refere aos fatores que diferem as pessoas ligeiramente felizes daquelas moderadamente e extremamente felizes.

DeNeve e Cooper (1998) sugeriram que a importância dada para a extroversão em relação ao bem-estar subjetivo tem sido exagerada em revisões realizadas anteriormente sobre o tema, enquanto muitos traços de personalidade pouco examinados mereceriam uma atenção especial. Ao mesmo tempo, os autores indicaram que não podiam concluir que a personalidade fosse a única variável importante para o bem-estar subjetivo, embora autores como Brebner, Donaldson, Kirby e Ward (1995) afirmassem que a estrutura básica de personalidade forma a base para a pessoa ser caracteristicamente feliz ou não.
Buscando definir felicidade, é comum a associação entre a freqüência e a intensidade de emoções agradáveis, ou seja, consideram-se mais felizes as pessoas que se apresentam intensamente felizes a maior parte do tempo. Diener (2000) abordou que experimentar emoções agradáveis a maior parte do tempo, e não experimentar emoções desagradáveis freqüentemente, mesmo se as emoções agradáveis forem apenas leves, já é um fato suficiente para altos relatos de felicidade.

Lyubormirsky (2001) mostrou que a felicidade pode realmente estar'em nossas cabeças', como a teoria popular tem afirmado há tanto tempo. Pessoas felizes apreciariam o que elas já têm, sem se prender àquilo que não têm.

Finalmente, o bem-estar subjetivo é associado à saúde e à longevidade, embora os caminhos ligando essas variáveis estejam longe de inteira compreensão (Diener \& Seligman, 2004). Seligman (2004, p.55) trouxe que "a emoção positiva funciona como previsão de saúde e longevidade, que são bons indicadores de reservas físicas".

\section{Estudos já realizados envolvendo o bem-estar subjetivo}

Diferentes estudos envolvendo o bem-estar subjetivo já foram realizados, principalmente fora do Brasil. Alguns estão descritos nos parágrafos abaixo, assim como os resultados obtidos.

Brebner et al. (1995) investigaram as relações existentes entre medidas de felicidade e personalidade. Para isso contaram com a participação de 95 estudantes voluntários. Seus resultados indicaram que a estrutura básica de personalidade delineia a tendência de uma pessoa ser caracteristicamente feliz ou não. O neuroticismo mostrou-se negativamente associado a medidas de felicidade, enquanto a extroversão mostrou-se positivamente relacionada a elas.

Já Vitters $\varnothing$ e Nilsen (2002) almejavam analisar e explorar a estrutura do bem-estar subjetivo, neuroticismo e extroversão e comparar os efeitos dos níveis do neuroticismo e da extroversão como preditores do bem-estar subjetivo. Através de uma população de 461 participantes adultos da Noruega, encontraram que o bem-estar subjetivo parece ser sustentado por três dimensões - a satisfação de vida, o afeto positivo e o afeto negativo - e também perceberam que o neuro- 
ticismo explicaria oito vezes mais a variância no bem-estar subjetivo do que a extroversão.

Lyubomirsky e Lepper (1999) realizaram estudos com um total de 2.732 participantes, procurando validar uma medida global de felicidade subjetiva. Participaram do estudo estudantes universitários dos Estados Unidos e da Rússia. Seus resultados indicaram consistência interna para os itens do instrumento, assim como uma correlação significativa quando avaliaram o teste-reteste.

Danner, Snowdon e Friesen (2001) trouxeram em seu estudo com freiras uma forte associação entre a emoção positiva nas autobiografias escritas e a longevidade observada seis décadas depois.

Uma interessante explicação para a relação entre bem-estar subjetivo, saúde e longevidade seria o fato de que o afeto positivo estaria relacionado a perfis favoráveis de funcionamento em diversos sistemas biológicos e poderia, dessa maneira, ser relevante ao reduzir o risco de desenvolvimento de doenças físicas (Steptoe, Wardle \& Marmot, 2005).

Um estudo de Cha (2003) buscou examinar o bem-estar subjetivo de 350 estudantes universitários da Coréia, assim como sua relação com construtos de personalidade, auto-estima, auto-estima coletiva e otimismo. A análise fatorial das medidas de bem-estar subjetivo indicou três fatores: satisfação de vida, afeto positivo e afeto negativo. Os estudantes mostraram resultados semelhantes aos de outros países, contradizendo os estudos de Diener, Suh, Smith e Shao (1995) e os estudos de Pavot e Diener (1993), que indicavam que os estudantes coreanos apresentavam valores mais baixos referentes à satisfação de vida e bem-estar afetivo quando comparados a estudantes de outras nações. Além disso, todos os construtos referentes à personalidade mostraram-se significativamente correlacionados com satisfação de vida, afeto positivo e afeto negativo.

Em seu artigo, Abdel-Khalek (2004) avaliou a felicidade de 141 estudantes Kuwaitianos, e encontrou que as médias de felicidade apresentadas por eles pareceram ser baixas quando comparadas com as apresentadas por estudantes de outros países.

Lever (2004) teve como objetivo comparar o bem-estar subjetivo de três grupos socioeconômicos - extremamente pobres, moderadamente pobres e não pobres. Para isso, contou com a participação de 918 participantes residentes no México. Seus resultados 516 indicavam diferenças estatísticas significativas em todos os fatores do bem-estar subjetivo em relação ao grupo socioeconômico a que os indivíduos pertenciam. E, por fim, a pesquisadora também encontrou diferenças relacionadas a sexo e idade.

No Brasil, poucos estudos sobre o bem-estar subjetivo foram realizados. Arteche e Bandeira (2003) focaram seu trabalho em avaliações sobre o bem-estar subjetivo de 193 adolescentes divididos em três grupos: o primeiro formado por 58 adolescentes trabalhadores em regime regular; o segundo por 58 adolescentes trabalhadores em regime educativo, e o terceiro por 77 adolescentes que não trabalhavam. Os resultados indicaram bons níveis de bem-estar nos três grupos, e mostraram que o trabalho na adolescência pode ser positivo, principalmente para os adolescentes que realizam um trabalho educativo.

Albuquerque e Tróccoli (2004) desenvolveram um instrumento para mensurar a satisfação com a vida, o afeto positivo e o afeto negativo, sendo esses os três componentes mais importantes do bem-estar subjetivo, segundo os autores. Um total de 795 policiais civis do Distrito Federal participou do estudo. Por meio da análise dos componentes principais e da análise fatorial, os autores encontraram em seu instrumento de 69 itens os três fatores esperados (satisfação com a vida, afeto positivo e afeto negativo), explicando 44,1\% da variância total do constructo.

Percebe-se, assim, a importância do desenvolvimento de outros estudos que abordem a importância do bem-estar subjetivo tanto para conhecer mais profundamente o tema como para adequá-lo à realidade brasileira.

\section{Considerações Finais}

A Psicologia Positiva surge como um inegável campo de estudos e como uma nova perspectiva para a Psicologia. O estudo das emoções positivas torna-se cada vez mais freqüente em estudos fora do Brasil. Cada vez mais estudos trazem contribuições acerca dos conteúdos da Psicologia Positiva, como, por exemplo, o bem-estar subjetivo.

Tendo em vista seu grande número de correlatos, o bem-estar subjetivo desperta grande interesse de pesquisadores empenhados em ampliar seus conhecimentos sobre as contribuições de aspectos positivos no 
dia-a-dia dos indivíduos. Abrem-se, dessa forma, as portas para um novo ramo de estudos da Psicologia que busca a compreensão das forças e virtudes humanas.

Poucos estudos foram realizados no Brasil até o presente momento sobre esse tema, indicando que grande parte da atenção dos pesquisadores brasileiros ainda está voltada para aspectos relacionados ao afeto negativo. Reside aí, então, uma interessante possibilidade de mudança de rumos.

Além de ser uma perspectiva atraente para aqueles que acreditam na importância do afeto positivo, estudos envolvendo o bem-estar subjetivo abrem as portas para novas contribuições que busquem o entendimento do ser humano a partir de suas potencialidades, e não apenas de suas fraquezas, como acontece tão fortemente até o momento.

\section{Referências}

Abdel-Khalek, A. M., \& Bandeira, D. R. (2004). Happiness among Kuwaiti college students. Journal of Happiness Studies, 5 (1), 93-97.

Albuquerque, A. S., \& Tróccoli, B. T. (2004). Desenvolvimento de uma escala de bem-estar subjetivo. Psicologia: Teoria e Pesquisa, 20 (2), 153-164.

Arteche, A. X. (2003). Bem-estar subjetivo: um estudo com adolescentes trabalhadores. Psico-USF, 8 (2), 193-201.

Bacon, S. F. (2005). Positive psychology's two cultures. Review of General Psychology, 9 (2), 181-192.

Burman, B., \& Margolin, G. (1992). Analysis of the association between marital relationships and health problems: an interactional perspective. Psychological Bulletin, 112 (1), 39-63.

Brebner, J., Donaldson, J., Kirby, N., \& Ward, L. (1995). Relationships between happiness and personality. Personality and Individual Differences, 19 (2), 251-258.

Cha, K. H. (2003). Subjective well-being among college students. Social Indicators Research, 62 (1), 455-477.

Danner, D. D., Snowdon, D. A., \& Friesen, W. V. (2001). Positive emotions in early life and longevity: findings from the nun study. Personality Processes and Individual Differences, 80 (5), 804-813.

DeNeve, K. M., \& Cooper, H. (1998). The happy personality: traits and a meta-analysis of 137 Personality Subjective Well-Being. Psychological Bulletin, 124 (2), 197-229.

Diener, E. (2000). Subjective well-being: the science of happiness and a proposal for a national index. American Psychologist, 55 (1), 34-43.

Diener, E., \& Biswas-Diener, R. (2002). Will money increase subjective well-being? A literature review and guide to needed research. Social Indicators Research, 57 (2), 119-169.
Diener, E., \& Seligman, M. E. P. (2004). Beyond money: toward an economy of well-being. Psychological Science in the Public Interest, 5 (1), 1-31.

Diener, E., Suh, E., Smith, H., \& Shao, L. (1995). National differences in reported subjective well-being: why do they occur? Social Indicators Research, 34 (1), 7-32.

Diener, E., Suh, E., \& Oishi, S. (1997). Recent findings on subjective well-being. Indian Journal of Clinical Psychology, $24(1), 25-41$.

Francis, L. J. (1999). Happiness is a thing called stable extraversion: a further examination of the relationship between the Oxford Happiness Inventory and Eysenck's dimensional model of personality and gender. Personality and Individual Differences, 26 (1), 5-11.

Joseph, S., Linley, P. A., Hardwood, J., Lewis, C. A., \& McCollam, P. (2004). Rapid assessment of well-being: The Short Deppression-Happiness Scale (SDHS). Psychology and Psychotherapy: Theory, Research and Practice, 77 (4), 463-478.

Lever, J. P (2004). Poverty and subjective well-being in México. Social Indicators Research, 68 (1), 1-33.

Lyubomirsky, S. (2001). Why are some people happier than others? The role of cognitive and motivational processes in well-being. American Psychologist, 56 (3), 239-49.

Lyubomirsky, S., \& Lepper, H. S. A. (1999). Measure of subjective happiness: preliminary reliability and construct validation. Social Indicators Research, 46 (2), 137-155.

Myers, D. G. (2000). The funds, friends, and faith of happy people. American Psychologist, 55 (1), 56-67.

Pavot, W., \& Diener, E. (1993). Review of the satisfaction with life scale. Psychological Assessment, 5 (2), 164-172.

Seligman, M. E. P., \& Csikszentmihalyi, M. (2000). Positive psychology: an introduction. American Psychologist, 55 (1), 5-14.

Seligman, M. E. P. (2004). Felicidade autêntica: usando a nova psicologia positiva para a realização permanente. Rio de Janeiro: Objetiva.

Seligman, M. E. P., Steen, T. A., Park, N., \& Peterson, C. (2005). Positive psychology progress: empirical validation of interventions. American Psychologist, 60 (5), 410-421.

Sheldon, K. M., \& King, L. (2001). Why positive psychology is necessary. American Psychologist, 56 (3), 216-217.

Simonton, D. K., \& Baumeister, D. R. F. (2005). Positive psychology at the summit. Review of General Psychology, 9 (2), 99-102.

Steptoe, A., Wardle, J., \& Marmot, M. (2005). Positive affect and health-related neuroendocrine, cardiovascular, and inflammatory processes. PNAS, 102 (18), 6508-6512.

Vittersø, J., \& Nilsen, F. (2002). The conceptual and relational structure of subjective well-being, neuroticism, and extraversion: once again, neuroticism is the important predictor of happiness. Social Indicators Research, 57 (1), 89-118.

Recebido em: 27/1/2006

Versão final reapresentada em: 26/10/2006

Aprovado em: 20/12/2006 
